\title{
Sub-sampled Fabry-Perot photoacoustic scanner for fast 3D imaging
}

Nam Huynh

Felix Lucka

Edward Zhang

Marta Betcke

Simon Arridge

Paul Beard

Ben Cox 


\title{
Sub-sampled Fabry-Perot photoacoustic scanner for fast 3D imaging
}

\author{
Nam Huynh, Felix Lucka ${ }^{1}$, Edward Zhang, Marta Betcke ${ }^{1}$, Simon Arridge ${ }^{1}$, Paul Beard, Ben Cox \\ Department of Medical Physics and Biomedical Engineering \\ Department of Computer Science ${ }^{1}$, \\ University College London, WC1E 6BT, UK
}

\begin{abstract}
The planar Fabry Perot (FP) photoacoustic scanner provides exquisite high resolution 3D images of soft tissue structures for sub-cm penetration depths. However, as the FP sensor is optically addressed by sequentially scanning an interrogation laser beam over its surface, the acquisition speed is low. To address this, a novel scanner architecture employing 8 interrogation beams and an optimised sub-sampling framework have been developed that increase the data acquisition speed significantly. With a $200 \mathrm{~Hz}$ repetition rate excitation laser, full $3 \mathrm{D}$ images can be obtained within 10 seconds. Further increases in imaging speed with only minor decreases in image quality can be obtained by applying sub-sampling techniques with rates as low as $12.5 \%$. This paper shows $3 \mathrm{D}$ images reconstructed from sub-sampled data for an ex vivo dataset, and results from a dynamic phantom imaging experiment.
\end{abstract}

Keywords: Photoacoustic tomography, Fabry Perot sensor, compressed sensing

\section{INTRODUCTION}

The Fabry-Perot ultrasound sensor can provide high resolution 3D photoacoustic images of tissue structures [1-4]. By interrogating the sensor with a scanning focussed laser beam, it is possible to synthesise arrays of many tens of thousands of points with small element sizes. However, the combination of the sequential nature of the sensor read-out and the low pulse repetition frequency (PRF) of most optical parametric oscillator (OPO) based excitation laser systems results in long acquisition times. For example, a typical 2D scan comprising 10,000 A-lines over a $10 \mathrm{~mm} \times 10 \mathrm{~mm}$ scan area takes approximately 4 minutes using an excitation laser with a PRF of $50 \mathrm{~Hz}$.

Significant reductions in acquisition time can be achieved by scanning multiple interrogation beams simultaneously in order to parallelise the detection. Using an 8 beam scanner and a $200 \mathrm{~Hz}$ excitation laser, 3D high resolution images (100 x 100 detection points) can be acquired within 10 seconds. In addition, video rate acquisition (17fps) is achievable for 2D images (100 detection points) [5]. To further increase 3D image acquisition speed, an optimised sub-sampling strategy has been developed that allows fewer measurement points to be used without a significant loss of image quality. It is anticipated that the significant increase in acquisition speed provided by this new scanner design will pave the way to fast 3D clinical imaging of dynamic physiological events with unprecedented image resolution.

\section{MULTI-BEAM FABRY PEROT PHOTOACOUSTIC SCANNER}

\subsection{Experimental setup}

Figure 1 shows a schematic of the 8-beam scanner. The output of a fibre-coupled 1550nm interrogation source (Santec TSL-550) is amplified by an $80 \mathrm{~mW}$ fibre amplifier (Nettest Fiberamp-BTF). The amplifier output is then split equally between the 8 fibres connected to an 8-beam bundle. A photodiode transimpedance amplifier configuration with DC- and

Photons Plus Ultrasound: Imaging and Sensing 2017, edited by Alexander A. Oraevsky, Lihong V. Wang, Proc. of SPIE Vol. 10064, 100641Y · @ 2017 SPIE · CCC code: 1605-7422/17/\$18 · doi: 10.1117/12.2250868 
AC-coupled outputs is connected to the return port of each circulator in order to detect the reflected beams. The DCcoupled outputs are connected to a 200kS/s 16-bit analog-to-digital (A/D) card within the PC and used to record the interferometer transfer function (ITF). The AC-coupled outputs are connected to an 8 -channel $60 \mathrm{MHz}$ digitizing card and used to record the time varying reflected optical power modulation produced by the incident acoustic wave. An X-Y optical scanner comprising a pair of mutually orthogonal closed loop galvanometer mirrors was used to scan the 8-beams across the FP sensor head. The scanner is mounted on an articulated arm that eases the imaging capability to different parts of a human body, e.g. neck, foot, arm.

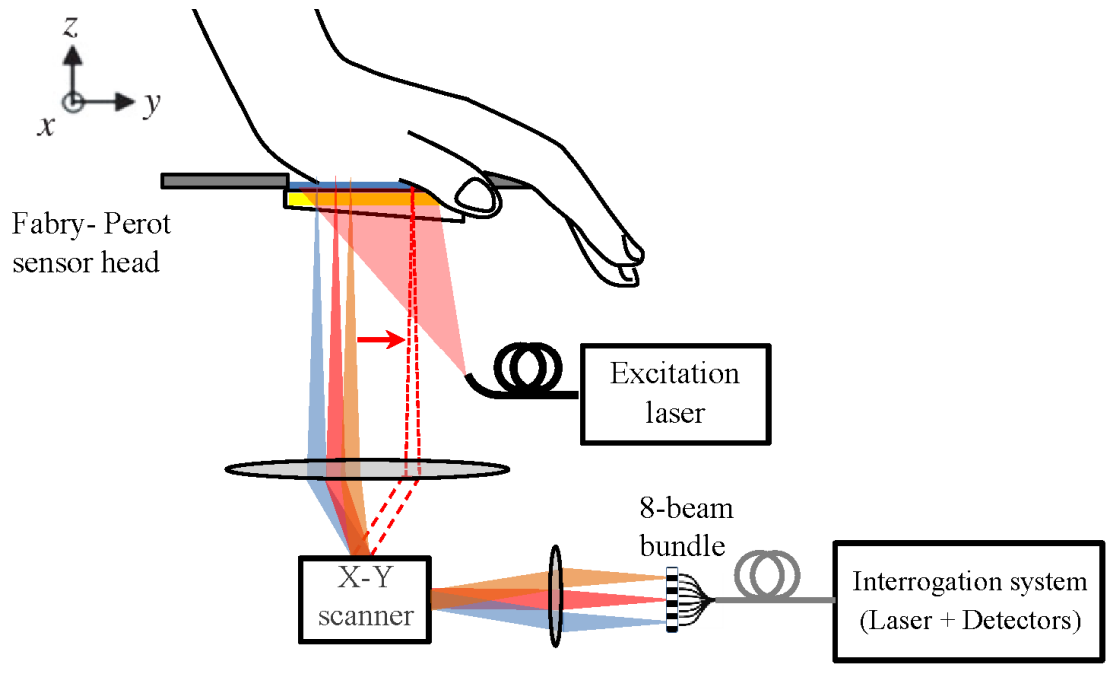

Figure 1 Schematic of the multi-beam scanner

The beam spacing at the sensor surface is approximately $315 \mu \mathrm{m}$. In order to achieve a higher scanning resolution, an interleaved scanning scheme was applied, e.g. 2 interleaved positions were used in all the experiments on this study to achieve a spatial sampling interval of $105 \mu \mathrm{m}$. Two excitation lasers were used in this study. The first one was a customdesigned, table-top Innolas OPO $(660 \mathrm{~nm}-1300 \mathrm{~nm})$ operating at a PRF of $200 \mathrm{~Hz}$ and providing pulse energies in the range $10-15 \mathrm{~mJ}$ depending on wavelength. The second excitation source was a fiber-coupled, $30 \mathrm{~Hz}$, OPO laser system (SpitLight 600, InnoLas Laser GmbH, Krailling, Germany). The in vivo imaging used the former excitation laser while the sub-sampling dynamic imaging study employed the latter.

\subsection{Sub-sampling method on Fabry Perot sensor}

Sub-sampled PAT using a Fabry Perot sensor was discussed extensively in [6]. In many situations, the spatio-temporal complexity of the absorbing tissue structures is rather low, and therefore data recorded in a conventional, regularly sampled, fashion is highly redundant in both space and time. It is possible, therefore, to speed up the data acquisition without a loss of image quality by exploiting this redundancy and measuring a subset of the data chosen in such a way as to maximise its non-redundancy. One way to achieve this is to sample the photoacoustic field at the smaller number of randomly chosen points $\left(y_{j}, z_{j}\right), j=1, \ldots, M_{c}, M_{c}<M$, which would yield an acceleration factor of $M_{s u b}=M / M_{c}$, where $M$ is the total number of points in the scanning area. A forward PAT model for the measured data $f^{c}$ can be written as:

$$
f^{c}=C \bar{p}+\varepsilon=C A p_{0}+\varepsilon,
$$

where $\bar{p}$ is the pressure on the detection plane, $C$ is the sampling operator (including the sub-sampling), $A$ is the forward operator modelling the acoustic propagation of the initial pressure $p_{0}$ and $\varepsilon$ accounts for additive measurement noise. To reconstruct $p_{0}$ from the sub-sampled data $f^{c}$, we use a variational regularisation approach:

$$
p_{\lambda}=\operatorname{argmin}_{p \geq 0}\left\{\frac{1}{2}\left\|C A p_{0}-f^{c}\right\|_{2}^{2}+\lambda T V(p)\right\},
$$


where $p_{\lambda}$ is the regularised solution and $T V(p)$ measures the total variation (TV) of $p$. While the first term in Eq. 2 measures the misfit between the measured and predicted data, the TV functional suppresses unwanted features like noise and artefacts in the regularised solution $p_{\lambda}$; the regularisation parameter $\lambda>0$ controls the balance between both terms. More details can be found in [6]. In the dynamic case, where the spatio-temporal complexity of imaging targets is changing significantly during data acquisition time, one can apply (2) to each frame of recorded data. However, a further increase of image quality (or dynamic frame rate) can be achieved by exploiting the temporal redundancy of the data. For this, we couple the spatial image reconstruction with a regularised motion estimation based on the optical flow equation [7]:

$$
P_{(\alpha, \beta, \gamma)}=\operatorname{argmin}_{P \geq 0}\left\{\min _{V} \frac{1}{2} \sum_{t=1}^{T}\left\|C_{t} A p_{t}-f_{t}^{c}\right\|_{2}^{2}+\alpha T V\left(p_{t}\right)+\beta T V\left(v_{t}\right)+\frac{\gamma}{2}\left\|\left(p_{t+1}-p_{t}\right)+\left(\nabla p_{t}\right) \cdot v_{t}\right\|_{2}^{2}\right\},
$$

where $P_{(\alpha, \beta, \gamma)}$ is the whole sequence of regularized 3D PA images, the sub-script $t$ refers to a single-frame quantity (e.g., $p_{t}$ is the $t$-th $3 \mathrm{D}$ volume in the $4 \mathrm{D}$ volume $P$ ), and $v_{t}$ denotes a $3 \mathrm{D}$ vector field describing the motion between images frames $p_{t}$ and $p_{t+1}$. Further details of this more sophisticated spatio-temporal reconstruction approach will be discussed in a forthcoming publication.

\section{RESULTS}

\subsection{Full sampling 3D in vivo imaging}

3D in vivo images of the subcutaneous vasculature in the human wrist and palm of a volunteer were obtained. The photoacoustic signals were mapped by scanning the 8 beams over an area of $10 \mathrm{~mm} \times 10 \mathrm{~mm}$ with steps of $105 \mu \mathrm{m}$ (on the wrist) and of $14 \mathrm{~mm} \times 14 \mathrm{~mm}$ (on the palm). The imaging times were approximately 10 seconds and 20 seconds, respectively. Figure $2 \mathrm{a}$ shows the hand resting on the FP sensor during experiments. Figure 2 shows maximum intensity projections (MIPs) of the 3D image data sets, colour-coded to depth. The excitation wavelength was set to $750 \mu \mathrm{m}$ for the wrist imaging and $860 \mu \mathrm{m}$ for the palm imaging. The $3 \mathrm{D}$ images were reconstructed from the detected signal using the $3 \mathrm{D}$ k-space algorithm [8].
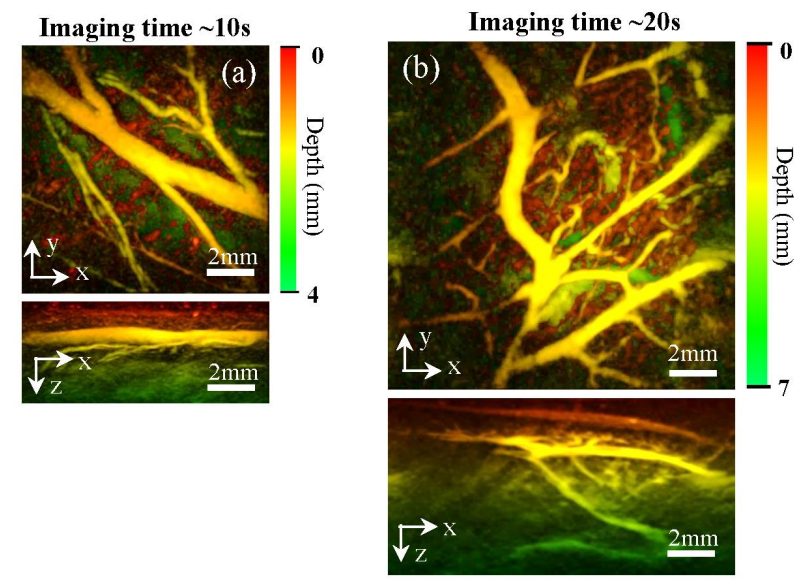

Figure 2 3D photoacoustic imaging of the human (a) wrist and (b) palm

\subsection{Sub-sampling PAT imaging: Static study}

The fully sampled dataset of the palm shown in section 3.1 was used to demonstrate the idea of sub-sampling imaging and reconstruction. A full data reconstruction using Eq. (2) is shown in figure 3a. Then, the full dataset was artificially sub-sampled using a 'random' pattern for the 8-beam configuration. The sampling pattern was designed to have the minimum number repeated locations, (locations at which the data may be reacquired by a different channel of the multibeam bundle). An example of the $25 \%$ sampling pattern is shown in figure $3 \mathrm{~b}$. Figure $3 \mathrm{c}$ shows the corresponding reconstructed images using (2). The images obtained from sub-sampled data show that all the main image features are retained with high resolution and SNR. The imaging time would have been just $\sim 5$ seconds for this dataset if only the sub-sampled data had been acquired directly. 
Full data reconstruction
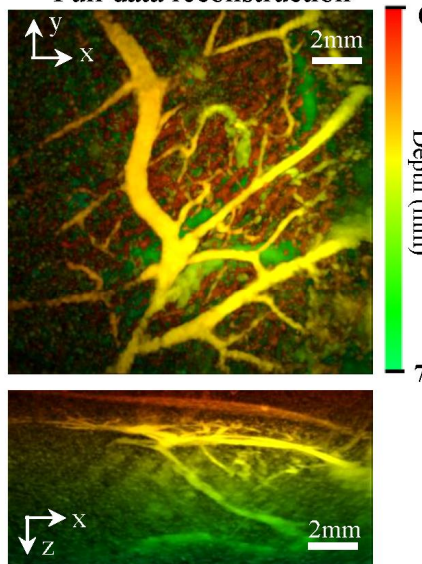

(a)

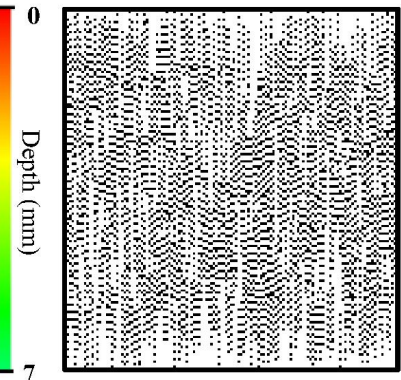

(b)

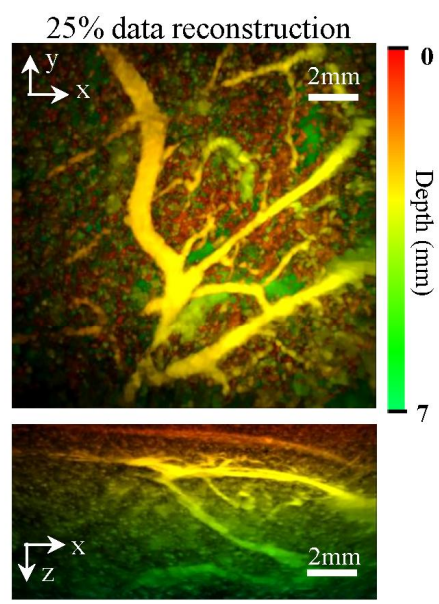

(c)

Figure 3 3D ex vivo photoacoustic imaging of the mouse: (a) full-data reconstruction using (2), (b) $25 \%$ sampling pattern for 8-beam system, (c) 8-beam subsampling reconstruction using (2) with $25 \%$ data set

\subsection{Sub-sampling PAT imaging: Dynamic study}

In this study, a dynamic phantom was imaged using the sub-sampling approach. The phantom was a low density polythene tube (ID 580 $\mu \mathrm{m} /$ OD $960 \mu \mathrm{m}$ Portex tubing TU3662 Scientific Supplies) containing a copper sulphate pentahydrate $\left(0.5 \mathrm{M} \mathrm{CuSO}_{4} .5 \mathrm{H}_{2} \mathrm{O}\right)$ solution. The tube was tied into a knot and immersed in a $1 \%$ intra-lipid solution. The tube was securely held at one end while the other end was attached to a motorised stage. The stage was controlled to move in one direction to tighten the knot while the scanner was continuously measuring data. In order to avoid the biased wavelength tuning time, a FP sensor with good thickness uniformity within the $10 \mathrm{~mm} \times 10 \mathrm{~mm}$ scanning area was used. Hence, during the imaging course, only one biased wavelength was used. Figure 4a shows the experimental setup. The MIP images for full-data and sub-sampled reconstructions using approaches Eqs (2) and (3) are shown in figure 4b. With the full data, image blurring is clearly present (indicated by white arrows) due to the motion of the phantom during the acquisition of a whole data set. For the images obtained using Eq. (2) there is less blurring but artefacts (indicated by red arrows) are still present. Also, the motion is not smooth between sequential images (although this cannot be seen in this one snapshot). Using Eq. (3) for the image reconstruction both further reduces artefact and leads to a smooth motion of the tightening knot.

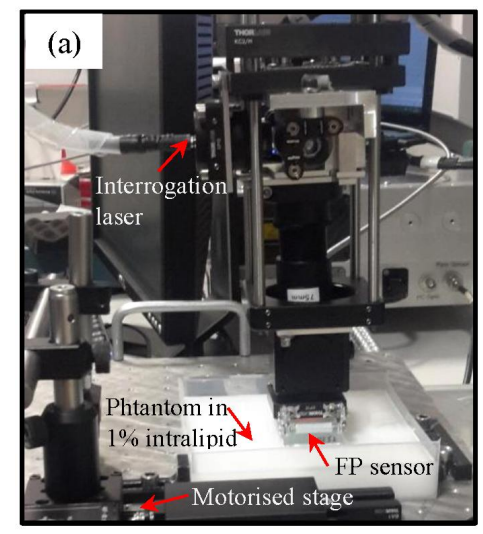

(b)
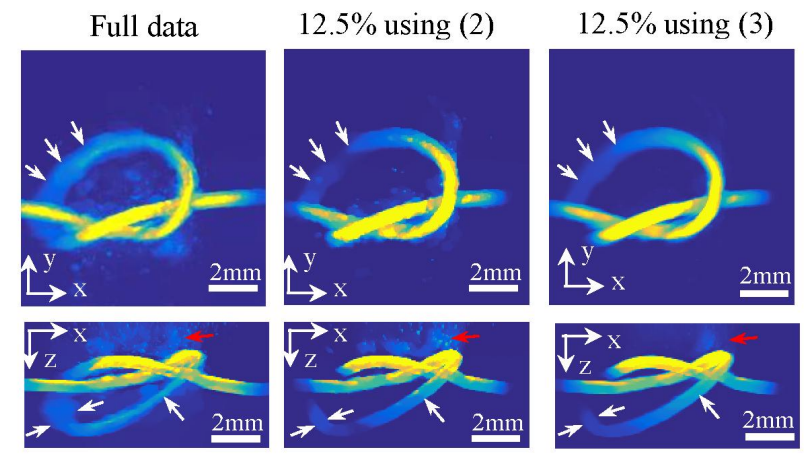

Figure 4 Dynamic PA imaging with subsampling patterns designed for 8-beam system: (a) experimental setup, (b) reconstructed MIPs images of a phantom. The arrows show regions that 'image blurring' (white arrows) and 'artefacts' (red arrow) are improved by using different reconstruction methods 


\section{CONCLUSION}

A multi-beam scanner architecture that employs 8 equally spaced interrogation beams in order to parallelise the FP sensor read-out has been developed. Together with the high PRF of the excitation laser $(200 \mathrm{~Hz})$, this new system enabled significant reductions in image acquisition time to be achieved, e.g. 3D images can be acquired in 10 seconds. Further improvement can be achieved by applying a sub-sampling technique. Our study shows good image reconstructions with a sub-sampling rate as low as $25 \%$ for in the static study and $12.5 \%$ for the dynamic imaging study using variational image reconstruction techniques.

\section{ACKNOWLEDGEMENTS}

The authors acknowledge support from the Engineering and Physical Sciences Research Council (EP/K009745/1), UK and European Union project FAMOS (FP7 ICT, Contract 317744). The authors are grateful for the support of Christian Menhard of Innolas $\mathrm{GmBH}$ who developed the $200 \mathrm{~Hz}$ OPO laser system.

\section{REFERENCES}

[1] Jathoul AP, Laufer J, Ogunlade O, Treeby B, Cox B, Zhang E, Johnson P, Pizzey AR, Philip B, Marafioti T, Lythgoe MF, Pedley RB, Pule MA and Beard P, "Deep in vivo photoacoustic imaging of mammalian tissues using a tyrosinasebased genetic reporter", Nature Photonics 9, 239-246 (2015)

[2] Zhang E., Laufer J., \& Beard P., "Backward-mode multiwavelength photoacoustic scanner using a planar Fabry-Perot polymer film ultrasound sensor for high-resolution three-dimensional imaging of biological tissues", Appl. Opt. 47, 561$577(2008)$

[3] Laufer J., Norris F., Cleary J., Zhang E., Treeby B., Cox B., Johnson P., Scambler P., Lythgoe M., Beard P., "In vivo photoacoustic imaging of mouse embryos", J Biomed Opt. 17(6), 061220 (2012)

[4] Beard P., "Biomedical photoacoustic imaging", Interface Focus, 1(4), pp.602-631(2011)

[5] Huynh N, Ogunlade O, Zhang E, Cox B, Beard P. "Photoacoustic imaging using an 8-beam Fabry-Perot scanner". Proc. of SPIE. 9708(97082L-1) (2016).

[6] Arridge S, Beard P, Betcke M, Cox B, Huynh N, Lucka F, Ogunlade O and Zhang E "Accelerated high-resolution photoacoustic tomography via compressed sensing", Phys. Med. Biol. 61 8908-8940 (2016)

[7] Burger M, Dirks H, Schönlieb CB, "A Variational Model for Joint Motion Estimation and Image Reconstruction", ArXiv e-prints 1607.03255 (2016)

[8] Koestli, K. P., Frenz, M., Bebie, H., Weber, H. P., Köstli, K. P., Frenz, M., ... Weber, H. P. “Temporal backward projection of optoacoustic pressure transients using fourier transform methods". Physics in Medicine and Biology, 46(7), 1863-72 (2001). 